\title{
Linx
}

Revue des linguistes de l'université Paris X Nanterre

$61 \mid 2009$

Entre rection et incidence : des constructions verbales atypiques?

\section{«Je pense donc... je dis. »}

Pour une syntaxe des (pré-)dicteurs

Dan Van Raemdonck

\section{OpenEdition}

\section{Journals}

Édition électronique

URL : http://journals.openedition.org/linx/1330

DOI : 10.4000/linx.1330

ISSN : 2118-9692

Éditeur

Presses universitaires de Paris Nanterre

Édition imprimée

Date de publication : 1 juin 2009

Pagination : $31-47$

ISSN : 0246-8743

Référence électronique

Dan Van Raemdonck, « « Je pense donc... je dis. » », Linx [En ligne], 61 | 2009, mis en ligne le 01 juin 2013, consulté le 30 avril 2019. URL : http://journals.openedition.org/linx/1330 ; DOI : 10.4000/

linx.1330

Département de Sciences du langage, Université Paris Ouest 


\title{
«Je pense donc ... je dis.» Pour une syntaxe des (pré-)dicteurs
}

\author{
Dan Van Raemdonck \\ Université Libre de Bruxelles - Centre de Linguistique - \\ Plateforme GRAMM-R
}

\section{Introduction}

Nous avons proposé (2002 et 2004) une analyse syntaxique des structures de discours rapporté, parmi lesquelles on retrouve des exemples du type Elle me dit qu'elle arrivera demain, Elle me dit : "J'arriverai demain » ou encore J'arriverai demain, me dit-elle. Pour chacune de ces trois structures nous avions dégagé une analyse spécifique : structure sous-phrastique déterminant du verbe de dire; structure de discours reproduit $(\Theta)$ déterminant du verbe de dire ; structure de discours re-produit en position phrastique, avec incise comme complément/déterminant de l'énonciation. Chacune de ces trois structures dénote un dégré différent d'intégration phrastique du discours cité.

La structure $\Theta$ vue comme marqueur de la complexité de l'énonciation (à côté de la complexité de la composante phrastique de l'énoncé, marquée par les sousphrases) nous a permis de questionner la place des verbes de dire dans la classe des recteurs faibles, dans la mesure où le déterminant d'un verbe de dire, dans le cas du discours re-produit, est bien régi par le verbe lui-même (il se pronominalisera en le) et apparait dans une certaine mesure comme selectionné par le verbe lui-même : un verbe de dire requiert comme déterminant un dit (ici le discours re-produit).

Nous essayerons de proposer une analyse systémique similaire pour les verbes prédicteurs, c'est-à-dire ceux qui précèdent le dire dans le modèle énonciatif du «moi-ici- 
maintenant, je perçois/ je conçois/je crois/ je pense et je dis que $p$ ». Nous nous limiterons ici à essayer d'inscrire dans notre modèle d'analyse phrastique les tournures pré-dictives construites avec le verbe penser et de faire la part entre le régi, le requis et le sélectionné.

\section{Rappel du modèle théorique}

Dans le cadre d'une syntaxe d'inspiration guillaumienne, nous avons proposé d'inscrire les fonctions syntaxiques dans un système ${ }^{1}$ où elles sont toutes définies à partir d'un même critère, l'incidence (relation entre un apport et un support de signification). À côté du support-sujet (que nous appellerons dorénavant noyau de phrase), caractérisé par une incidence interne, et des apports à des termes de la phrase (déterminants du nom, du verbe, de l'adjectif, de l'adverbe... ; prédicats de terme), caractérisés eux par une incidence externe du premier degré, on distingue encore des apports (déterminants ou prédicats; T3 dans le schéma ci-dessous) à des relations entre deux termes, apports morphologiquement divers (du mot à la (sous-)phrase), qui sont unifiés par la caractéristique qu'ils ont en commun : l'incidence externe du second degré. Dans ce système, on substitue à une représentation traditionnelle du type $\mathrm{T} 1 \leftarrow$ T2 (où T2 est dit déterminant ou prédicat de T1) une représentation bidimensionnelle et plus hiérarchisée du type (où T3 est dit déterminant ou prédicat de la relation entre T2 et T1) :

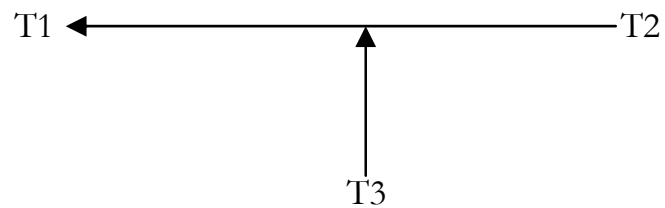

Les relations, supports potentiels d'apports (déterminants ou prédicats), sont elles aussi multiples et se rencontrent à des niveaux divers de la phrase, allant du niveau supérieur, la relation prédicative, au niveau inférieur, infrasyntagmatique ${ }^{2}$.

Couplé à la paire de mécanismes détermination et prédication, le critère de l'incidence permet d'établir une grille des fonctions de la phrase.

\footnotetext{
${ }^{1}$ Ce système de parties de discours ou fonctions double un autre système, de natures de mot, système de parties de langue organisé par le critère de l'extension emprunté à Wilmet (1986) et adapté par nos soins pour une distinction opérationnelle entre extension et incidence (voir notamment Van Raemdonck 1998 et 2010).

${ }^{2}$ Ce modèle s'inscrit dans le cadre plus large d'une syntaxe de type génétique. C’est ainsi que nous posons l'existence de deux types d'incidence (Van Raemdonck 2002) : une incidence effective, qui relie effectivement l'apport de signification à son support, et une incidence d'attente, qui préfigure et donc précède l'incidence effective, avant sa réalisation, dans la genèse de la phrase. Chaque terme ou chaque groupe constitué, avant même d'être effectivement mis en relation avec d'autres éléments, peut être déclaré en attente soit de support, soit d'apport (de détermination ou de prédication). Dans les schémas de la présente contribution, nous ferons l'économie de la prise en compte de ce double niveau d'incidence.
} 
Par détermination, nous entendons le mécanisme grammatical général par lequel un élément est rapporté à un autre élément ou à une relation et $1^{\circ}$ ) réduit l'extension ou donne une indication sur l'extensité 3 de l'élément déterminé, $2^{\circ}$ ) réduit l'extension de la relation sur laquelle il porte. Le déterminant se comporte comme un complément d'information. Nous parlerons donc de déterminants du nom, du pronom, du verbe, de l'adjectif, de l'adverbe, du connecteur prépositionnel..., ainsi que de déterminant de relation (relation prédicative, relation entre le déterminant et le noyau nominal, entre le déterminant et le noyau verbal...). Le groupe de mot(s) organisé et hiérarchisé qui réunit autour d'un support-noyau ses apports par le biais de relations de type déterminatif sera appelé groupe déterminatif (nominal si le noyau est un nom (GDN); verbal si le noyau est un verbe $(\mathrm{GDV}) \ldots)^{4}$. La relation de détermination est inscrite dans les schémas à l'aide d'une flèche simple.

La détermination est encore à l'œuvre lorsqu'il s'agit d'apporter de l'information non pas à la composante phrastique de l'énoncé, mais bien à la composante énonciation de celui-ci. Ainsi, Franchement dans Franchement, tu exagères sera-t-il considéré comme déterminant de l'énonciation. Non pas dans le sens où l'énonciation serait rectrice de quoi que ce soit, mais dans la mesure où ce déterminant est bien un complément d'information apporté à l'énonciation (sur la manière du dire en l'occurrence). Syntaxiquement, il s'agit d'un déterminant dont l'échéance est sur la relation prédicative, ciment de la phrase : l'énonciation n'a pas de place prédéfinie dans une phrase; elle est le bain dans lequel est plongé la phrase pour faire énoncé. L'apport sémantique emporté par ce déterminant est quant à lui reversé à la composante énonciation. Le déterminant de l'énonciation sera inscrit dans les schémas à l'aide d'une flèche triple aboutissant à la relation prédicative, doublée d'une flèche en pointillé, aboutissant dans le bain environnant qu'est l'énonciation ${ }^{5}$.

Par prédication, nous entendons le mécanisme grammatical général par lequel un élément est rapporté à un autre élément ou à une relation sans en réduire l'extension. Le prédicat se comporte comme un supplément d'information, car lorsqu'il intervient sur un terme de la phrase, il le fait une fois la détermination opérée et le groupe déterminatif constitué, soit après la clôture de celui-ci. Pour éviter la confusion avec la prédication première et constitutive de la phrase, lorsque ces mêmes mécanismes seront mis en œuvre sans l'intervention d'un verbe conjugué à un temps fini, nous parlerons de prédication seconde. Ce sera notamment le cas pour les appositions (nominales ou adjectives, en ce compris les relatives explicatives), pour certains apports adverbiaux, qui prédiquent sans l'intermédiaire d'un verbe et pour les attributs de déterminant. Le groupe de mot(s) organisé et hiérarchisé qui réunit autour d'un support-noyau ses apports par le biais de relations de type prédicatif sera appelé groupe prédicatif (GP1 pour la phrase, organisée autour de la prédication première ; GP1' pour la sous-phrase ; GP2 pour le groupe prédicatif dont le noyau a comme apport un

\footnotetext{
3 Sur la notion d'extensité (" quantité d'éléments auxquels un terme est effectivement appliqué »), voir notamment Wilmet (1986 et 19971, 20105).

${ }^{4}$ Nous avons recours au terme de groupe déterminatif, plutôt qu’à celui de syntagme, pour permettre l'opposition au terme groupe prédicatif (voir infra).

${ }^{5}$ Cela répond à l'objection émise par Gachet (à par.) concernant la possibilité qu'aurait l'énonciation de régir quelque complément que ce soit.
} 
prédicat second (P2), voir infra $)^{6}$. La relation de prédication est inscrite dans les schémas à l'aide d'une flèche double.

Ainsi, une phrase du type Franchement/Heureusement/Souvent, Pierre éprouve du plaisir dans son travail pourra être représentée par le schéma suivant ${ }^{7}$ :

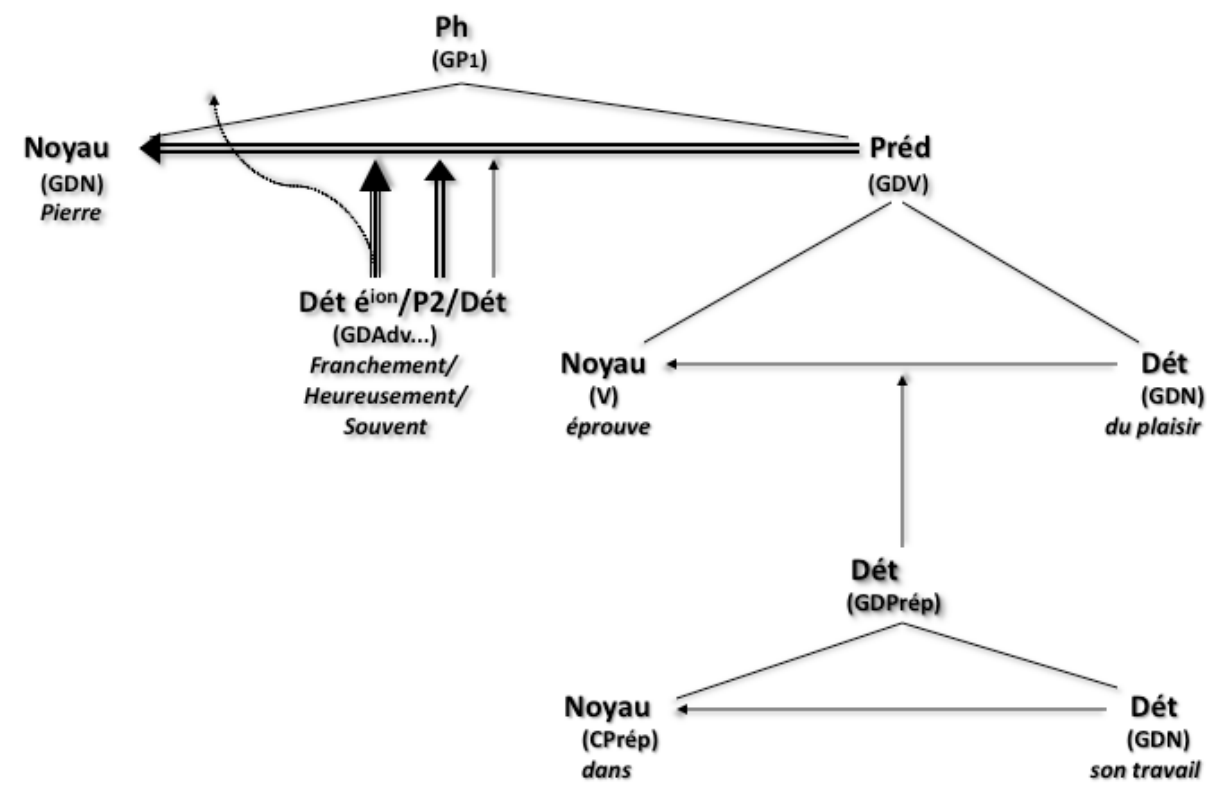

La portée intraprédicative des apports est révélée par le test de la négation. Si l'apport est sous la portée de la négation dite «descriptive », cela signifie qu'il est à l'intérieur du prédicat, dans la mesure où la négation ${ }^{8}$ clôt celui-ci'. Dans la version négative de la phrase ci-dessus (Franchement/Heureusement/Souvent, Pierre n'éprouve pas de plaisir dans son travail), éprowve, du plaisir et dans son travail seraient sous la portée de la

\footnotetext{
${ }^{6}$ Nous retrouvons, en affinant leur analyse, les options développées par Jespersen (1924) (nexus), repris par Eriksson (1993), et Hanon (1989) (constructions absolues). Voir également Van Raemdonck (2007).

${ }^{7}$ Pour des raisons d'économie, nous ne poussons pas jusqu'au bout la description des groupes dans les schémas de cette contribution.

8 Pour une argumentation, voir Van Raemdonck (2003).

${ }^{9}$ Le sujet est exclu de la portée de cette négation descriptive. Les apports à une relation qui sont hors de portée de la négation sont des apports à la relation prédicative première (entre le prédicat et le noyau de phrase). Ils ne seront dès lors pas régis par le verbe. Voir à ce sujet, notamment Van Raemdonck (1998b), pour la distinction entre les différents apports de la relation prédicative :

- Souvent, Pierre éprouve du plaisir dans son travail : déterminant de la relation prédicative.

- Heureusement, Pierre éprouve du plaisir dans son travail : prédicat second de la relation prédicative.

- Franchement, Pierre éprouve du plaisir dans son travail : déterminant de l'énonciation, avec échéance syntaxique sur la relation prédicative.
} 
négation; Franchement/Heureusement/Souvent et Pierre seraient hors de portée de la négation.

Le phrase $\mathrm{Ph}$ (GP1) est vue dans ce modèle comme un réseau récursif de structures intégratives, allant du groupe déterminatif (GDx (nominal, verbal...)) à la sous-phrase $\Delta\left(\mathrm{GP}^{\prime}\right)$, en passant par le groupe prédicatif second (GP2), qui se différencie des GP1 par le fait que la relation de prédication qui l'institue est une relation de prédication seconde et non première, c'est-à-dire dépourvue d'un verbe conjugué à un temps fini. En tableau :

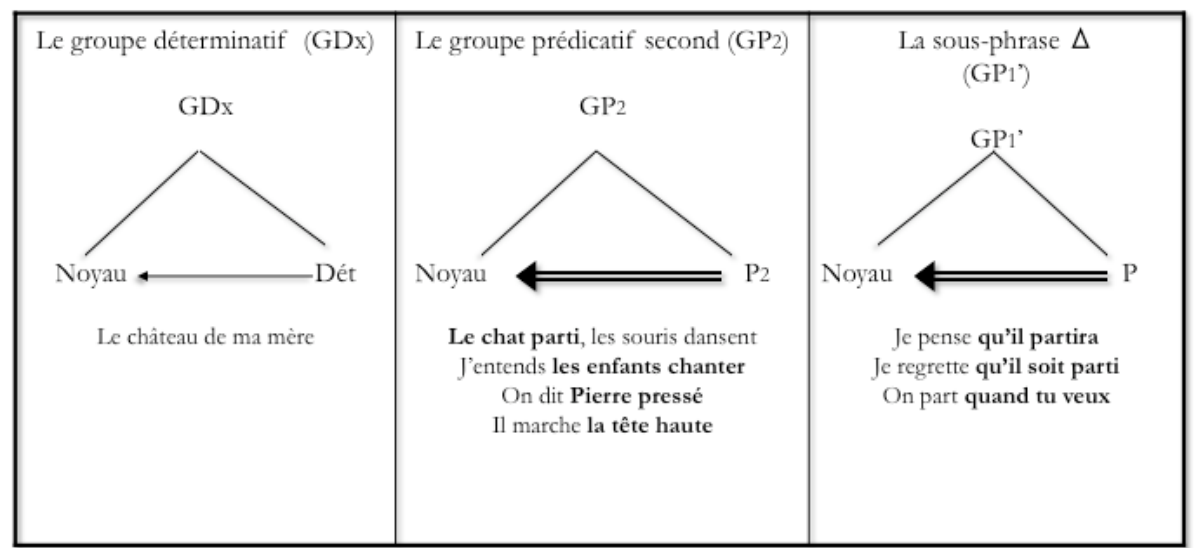

Enfin, la complexité, observée pour la composante phrastique (sous-phrase $\Delta$ ), peut également toucher l'énonciation par le biais de la structure $\Theta$ (ou discours reproduit (Van Raemdonck 2002, 2004 et 2007)), envisagée comme recouvrant des tournures où une séquence plus ou moins longue de discours direct est intégrée syntaxiquement, telle que prétendument ou supposément prononcée, c'est-à-dire sans réajustements formels, dans la position fonctionnelle d'un terme normal de phrase (morphème, élément de syntagme ou syntagme). En tableau :

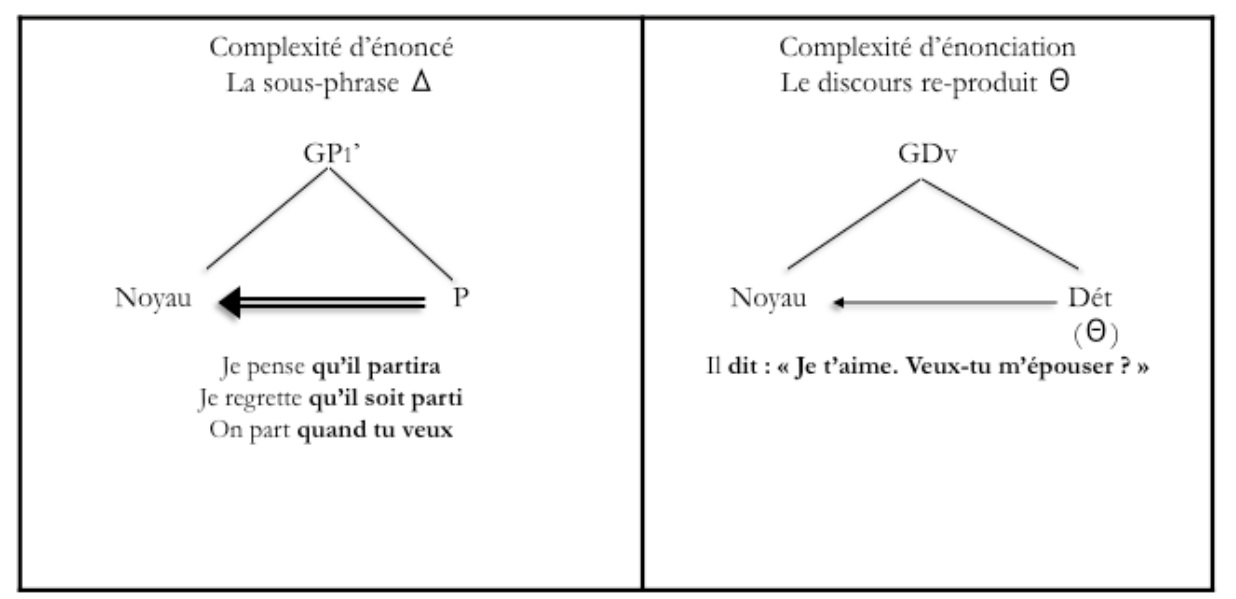




\section{La syntaxe du pré-dicteur penser}

Dans ce cadre théorique, comment envisager la syntaxe d'un verbe pré-dicteur comme penser. Faut-il y voir un verbe recteur plein, un verbe parenthétique (Urmson, 1952) ou un verbe recteur faible (Blanche-Benveniste 1989 et Blanche-Benveniste \& Willems 2007) ou encore un adverboïde (Apothéloz, 2003)?

\subsection{Parenthétique ou rection faible}

Les propriétés relevées par les auteurs (Urmson 1952 et Blanche-Benveniste 1989) pour caractériser un verbe parenthétique ou recteur faible sont les suivantes : il se trouve en tête de phrase suivi d'une sous-phrase déterminant du verbe introduite par que (Je pense que Pierre est déterminé) ; il se trouve tout aussi bien en incise, potentiellement après la séquence apparemment déterminant (Pierre est déterminé, je pense). Urmson (1952, p. 481) précise même que le verbe est à la première personne du présent. Blanche-Benveniste \& Willems (2007) ajoute une troisième propriété : la possibilité de trouver ce verbe en réponse derrière Oui (-C'est vrai.? - Oui, je pense).

L'idée serait que ces verbes ne disposeraient pas de leur sens plein. Il y aurait un effet atténuatif, modalisateur, qui devrait nous pousser à considérer la sous-phrase déterminant comme la vrai matrice. Et dès lors étudier une nouvelle forme de discordance entre sémantique et syntaxe, l'idée principale se trouvant dans la sous-phrase. Ajoutons à cela que, dans une perspective de rection faible, vu l'absence de pronom le pour pronominaliser la sous-phrase ou l'énoncé, le verbe serait très peu recteur ; qu'il serait donc difficile d'affirmer une relation de déterminant à noyau entre le verbe et la sousphrase en que ou entre l'énoncé et l'incise. Dès lors, la mutation en structure adverboïdale guette ces verbes (Apothéloz 2003). Gachet rend fort bien compte, dans le présent volume, des discussions sur les caractéristiques supposées, avérées ou non, desdits «recteurs faibles ». Nous ne pouvons donc que renvoyer le lecteur à cette contribution.

Pour notre part, il apparait important de redistribuer les cartes et de visualiser la manière dont s'organisent effectivement les relations syntaxiques entre les différentes prédications des séquences étudiées. Notre démarche se veut strictement, et modestement, syntaxique. L'idée que nous souhaitons avancer est qu'une description syntaxique cohérente permettrait de visualiser tous les effets de sens relevés. Dès lors la question reviendra moins à mesurer la force ou à la faiblesse de la rection du verbe (pré)-dicteur qu'à déterminer la fonction que l'énonciateur entend lui faire jouer dans son énoncé.

\subsection{Valence, rection et sélection}

Avant d'aller plus avant, il nous reste à recadrer un certain nombre de termes utilisés dans le cadre de la description de la complémentation verbale. On rencontre en effet les termes de valence (Tesnière 1965), d'éléments requis ou régis (Lazard 1994 et 
1998). La valence verbale intègre les actants du verbe, généralement limités au sujet et aux objets direct et indirect, à l'exclusion donc des circonstants ${ }^{10}$. Les éléments requis de Lazard reprennent les éléments dont la présence est exigée par le verbe, les éléments régis coiffant ceux dont la forme et la construction sont exigées par le verbe. Il n'entre pas dans notre intention de rediscuter ici de la pertinence de chacune de ces notions. Nous désirons néanmoins préciser ci-dessous la manière dont nous utilisons chacun des termes qui les recouvrent.

Selon nous, à y regarder de près, les termes de valence et d'éléments requis relèvent de la complétude sémantique du verbe. Ils pourront dès lors être utilisés conjointement, la valence du verbe reprenant les éléments requis par lui, indépendamment du contexte d'utilisation. Nous préférons, pour notre part, spécifier le terme de rection dans son versant syntaxique: serait régi l'élément qui échoit syntaxiquement à son terme recteur. Dans la phrase Franchement/Heureusement/Souvent, Pierre éprouve du plaisir dans son travail, à l'intérieur du GDV, seraient par exemple régis le déterminant du terme verbal (du plaisir) et le déterminant de la relation entre ce déterminant et le verbe lui-même (dans son travai), quand bien même ce déterminant de relation ne serait pas obligatoire, requis. En effet, le déterminant de relation (dans son travail) échoit à une relation (qui relie $d u$ plaisir à éprowve) qui échoit elle-même au verbe (éprowve $)^{11}$. Lorsque la forme ou le type de construction de l'apport seront exigés pour des raisons de cohérence ou de cohésion, nous dirons que l'élément est sélectionné.

Nous distinguons ainsi trois niveaux dans la relation au verbe: la valence correspond à l'épure de la complétude lexico-sémantique du verbe (l'élément requis en est l'élément constitutif) ; l'élément régi est celui dont, au niveau syntaxique, l'incidence échoit (jusqu')au verbe ; l'élément sélectionné est celui dont la forme et la construction sont exigés par le verbe.

\section{Description des structures}

\subsection{Selon elle, Pierre est déterminé}

Dans cette tournure, l'énonciateur assume son énoncé dans le cadre défini par le déterminant de la relation prédicative (Selon elle). Il prend donc en charge l'élément cité

\footnotetext{
${ }^{10}$ Les théories ultérieures de la valence verbale, dès les années 60 en Allemagne, développeront une autre vision, qui ramène certains circonstants de Tesnière dans le giron de la valence. Voir notamment, pour le français, la synthèse de Blanche-Benveniste (2001). Nous avons essayé d'affiner encore la discussion dans Van Raemdonck (2010).

${ }^{11}$ Un apport (déterminant ou prédicat (second)) peut être incident à un terme de la phrase ou à une relation entre deux termes. Si une relation, qui marque le processus de liaison entre deux termes, peut servir de support à un apport qui lui serait incident, peut-on dire qu'elle en est le terme recteur? Nous préférons faire le choix de considérer que le recteur doit être un terme de la phrase auquel sont rapportés syntaxiquement, directement ou indirectement, les apports caractérisés par une incidence externe du premier ou du second degré à l'intérieur du groupe dont ce terme est le noyau.
} 
Pierre est déterminé pour autant qu'il soit ramené à la personne que l'on « cite » ${ }^{12}$ via l'élément citant déterminant de relation. Ce déterminant de relation ne pose pas la question du Qui parle? (c'est bien l'énonciateur qui parle), mais celle du point de vue qu'adopte l'énonciateur pour assumer son énoncé. Contrairement à ce que l'on pourrait croire, malgré ce qui semble bien être une attribution du dire, il ne s'agit pas de déterminant de l'énonciation, mais de déterminant de l'énoncé. En effet, c'est bien l'énonciateur qui assume son énoncé, même s'il réduit la portée de sa vérité au point de vue évoqué. En schéma :

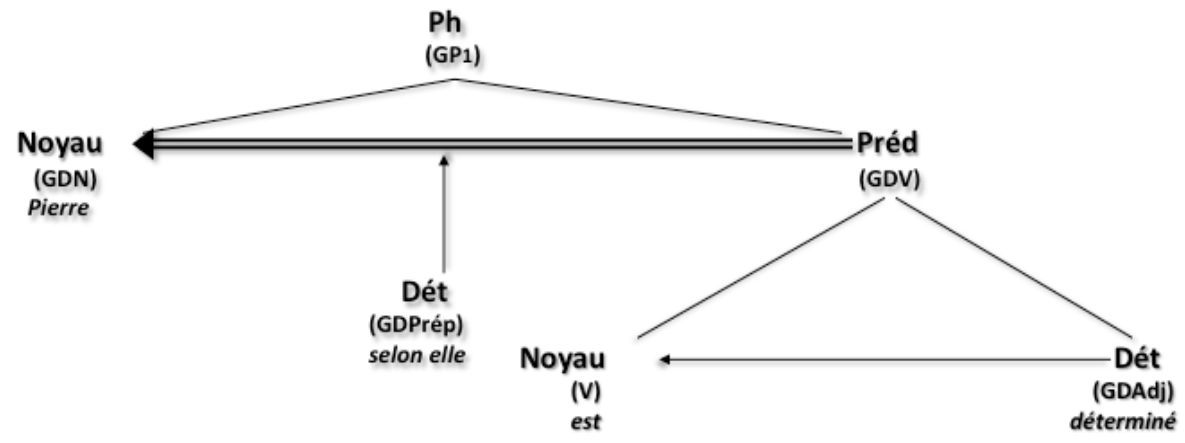

\subsection{Elle dit/pense que Pierre est déterminé.}

Figure prototypique du (pré-)discours indirect, cette tournure sépare nettement le discours cité Pierre est déterminé du discours citant Elle dit/pense. En effet, ce discours cité est en construction sous-phrastique, dépendant du verbe de dire, en fonction de déterminant du verbe. La phrase matrice se réduit à Elle dit/pense $\boldsymbol{\Delta}$, où $\Delta$ symbolise la sous-phrase dans la position fonctionnelle du groupe déterminatif nominal correspondant. Du point de vue de l'intégration syntaxique, l'enchâssement sous-phrastique à l'aide de que (enchâsseur et translateur) fait donc fonctionner le $\Delta$ comme un simple groupe déterminatif nominal déterminant du verbe. Ce déterminant $\Delta$ est par ailleurs pronominalisable en le (pronom neutre) : Elle le dit/pense. En schéma :

\footnotetext{
12 Nous reprenons aux théories du discours rapporté (Rosier 1999) les notions de discours citant et de discours cité, même si, dans le cas du verbe penser, il n'y a pas toujours dire ni discours. Nous les gardons néanmoins pour permettre le parallélisme entre verbe dicteurs et pré-dicteurs.
} 


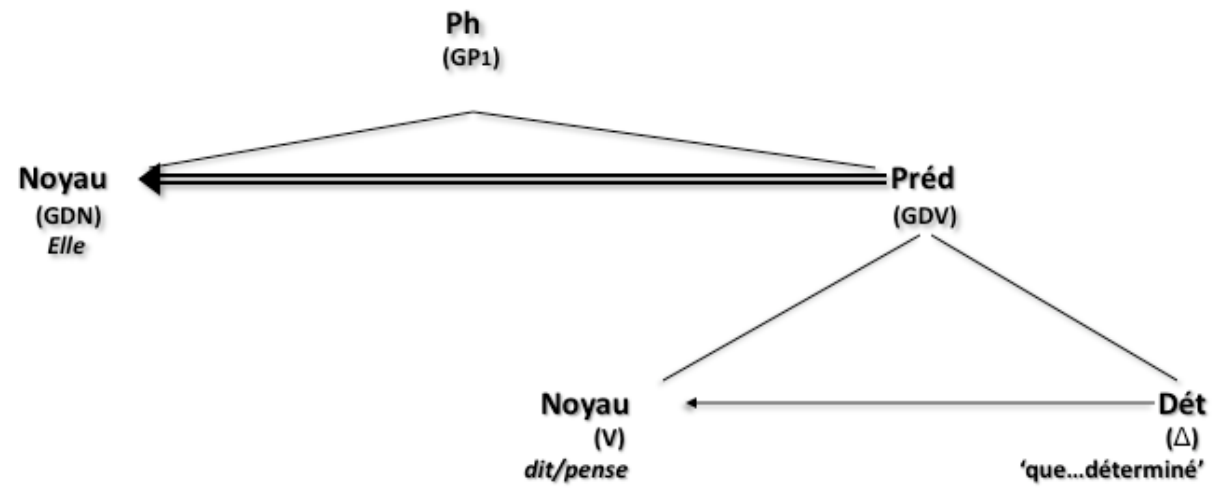

\subsection{Elle dit/pense : "Pierre est déterminé. "}

Dans cette tournure de (pré-)discours rapporté, le (pré-)discours direct, la coupure est plus nette encore entre discours citant et discours cité. Il y a clairement eu au départ deux énonciateurs, qui se maintiennent en apparence.

Nous avons proposé (Van Raemdonck 2002, 2004 et 2007) de prendre en considération des structures $\Theta$ (voir supra). Du point de vue de l'énonciation, cette séquence matrice a un seul énonciateur (le narrateur), quand bien même celui-ci aurait pris sous son aile énonciatrice un (pré-)discours d'autrui «thêtanisé ». Le déterminant du verbe dit/pense est un $\Theta$, qui devra être analysé par la suite. À un niveau d'analyse syntaxique hiérarchiquement inférieur, l'énonciateur de la séquence reprise sous ce $\Theta$ est Elle. Ce déterminant $\Theta$ est par ailleurs pronominalisable en le (pronom neutre) : Elle le dit/pense. En schéma :

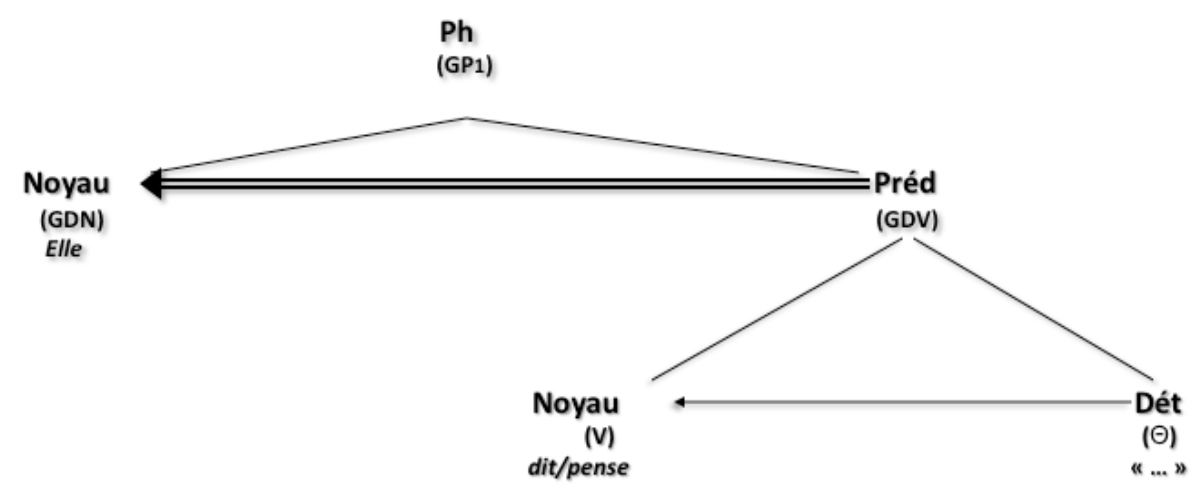

\section{4. "Je suis déterminée", dit-elle/pense-t-elle/qu'elle dit/pense.}

Dans cette structure, se croisent en apparence deux énonciateurs : l'un dans le discours cité de la matrice, qui dit le je du (pré-)discours direct ; l'autre dans le discours citant de l'incise insérée $(\Delta)$, qui dit le moi du narrateur. Dans ce cas-ci, ce déterminant 
répond bien à la question du Qui parle? Il s'agit bien d'un déterminant de l'énonciation (il en a toutes les caractéristiques, comme franchement : hors de la portée de la négation ; nonfocalisabilité à l'aide de c'est...que), et non d'un déterminant de l'énoncé. Il porte sur une relation prédicative qui ici se dérobe : Je suis déterminée constitue en fait un $\Theta$, en position phrastique avec prédication impliquée (en schéma, la relation prédicative se referme sur elle-même, comme un cercle), comme s'il s'agissait d'une interjection, où le noyau et le prédicat impliqués dans la relation prédicative ne sont pas directement exposés, explicités. Le verbe dit/pense de la sous-phrase est construit sans déterminant, ou avec un déterminant zéro: il semble difficile d'y insérer un pronom déterminant du verbe de type le. En schéma :

\section{$\mathrm{Ph}$}
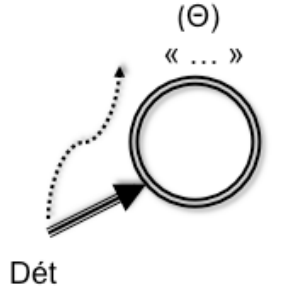

$(\Delta)$

dit-elle/pense-t-elle qu'elle dit/qu'elle pense

\subsection{Elle dit/pense Pierre déterminé.}

Dans ce cas, le déterminant du verbe est un groupe prédicatif second (GP2), que nous avons également appelé binôme (Van Raemdonck 2007), dans lequel le noyau (un groupe déterminatif nominal) Pierre se voit attribuer un prédicat second déterminé (il est par ailleurs impossible de se passer du prédicat second sans rendre la séquence caduque). Cette prédication seconde pourrait être le reliquat, après intégration phrastique, de la prédication d'origine Pierre est déterminé. Ce déterminant GP2 est par ailleurs pronominalisable en le (pronom neutre). En schéma :

$\mathrm{Ph}$

(GP1)

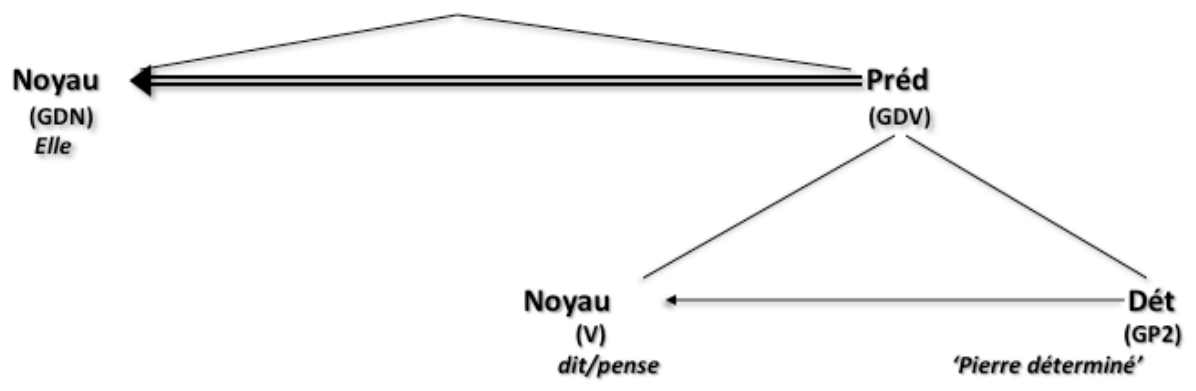




\subsection{Elle dit/?pense la détermination de Pierre.}

On se trouve ici devant un discours de type narrativisé (Rosier 1999). Il n'y a pas retranscription d'un discours ou d'une pensée complets, mais formulation synthétisée de leur teneur. Le groupe déterminatif nominal la détermination de Pierre est déterminant du verbe dit/?pense; il est totalement et normalement intégré à la syntaxe de la phrase. Cependant, avec le verbe penser, la seule acception envisageable ici est une acception non mobilisée jusqu'à présent : celle d'« embrasser (un objet, un concept) par la pensée ». Pour conserver en effet l'acception « estimer », "avoir pour opinion », présente dans les autres exemples, il faut en fait que le déterminant soit un procès/processus et non un objet. La pronominalisation, pour autant qu'elle soit possible dans le cas présent, se ferait en la et non en le. On peut donc en conclure que le parallélisme dire/penser n'est pas jusqu'auboutiste. En schéma :

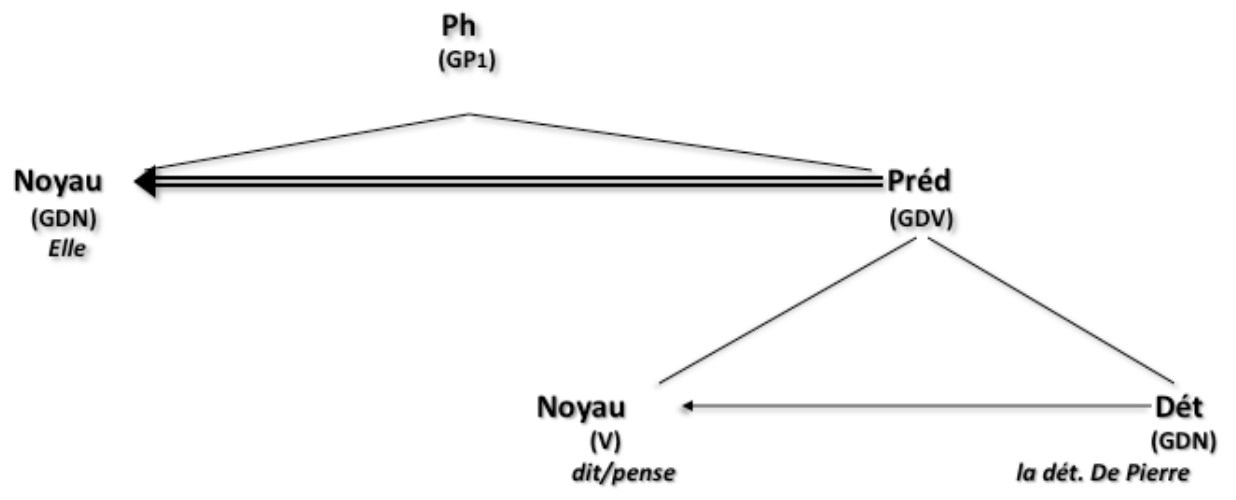

\subsection{Que Pierre est déterminé, elle le dit/pense.}

Dans cet exemple, le discours cité est pris en charge par la sous-phrase $\Delta Q u e .$. déterminé qui apparait ici comme un déterminant de l'énonciation, qui échoit syntaxiquement à la relation prédicative. Cette sous-phrase cadre le propos de l'énoncé. Elle est reprise par le pronom neutre le en position déterminant du verbe ; l'absence de ce pronom rendrait la phrase caduque. Si ce pronom le fait partie de la rection du verbe, la sous-phrase en est quant à elle exclue. Cette description vaut également lorsque la sous-phrase est postposée : Elle le dit/pense, que Pierre est déterminé. En schéma : 


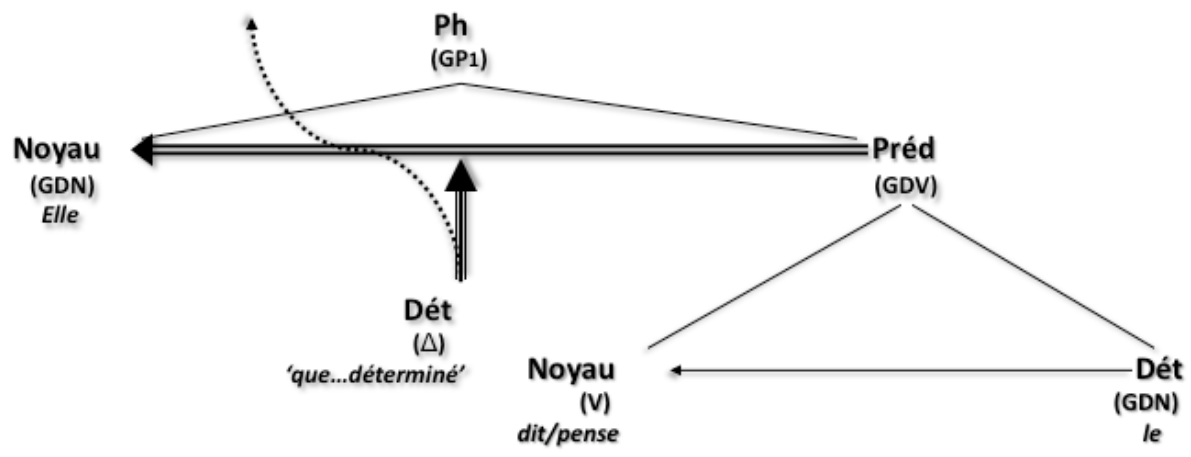

\section{8. "Pierre est déterminé ", elle le dit/pense.}

Une autre lecture-analyse que celle de l'exemple 4 est possible pour cet exemple, et même rendue plus plausible du fait de la reprise du discours cité par le pronom le. Il s'agirait de rapprocher cet exemple du précédent, et de considérer que "Pierre est déterminé » est un $\Theta$ qui occuperait la fonction décrite ci-dessus de déterminant de l'énonciation cadrant le propos. Ici également, la rection du verbe inclurait le pronom neutre mais pas le discours re-produit. En schéma :

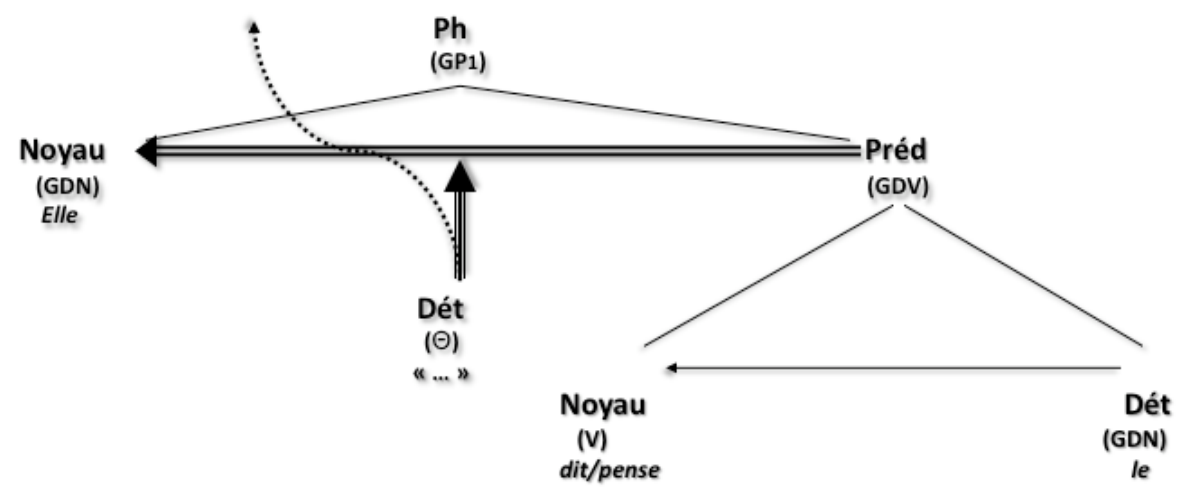

\subsection{Je pense que Pierre est déterminé}

Jusqu'à présent, nous avons étudié des structures où, à l'exception de l'incise déterminant de l'énonciation (exemple 4), le verbe (pré-)dicteur régissait un déterminant, fût-il sous la forme d'un pronom neutre. Cependant, si la question du «verbe recteur faible » affleure, c'est parce qu'il semble bien que, dans la mesure où tout dire présuppose un «moi-ici-maintenant, je perçois/ je conçois/je crois/ je pense et je dis que $p »$, une matrice avec verbe recteur du type penser apparait comme bien peu informative. Dès lors, la tentation de dégrader ce verbe est grande. Il ne serait plus 
verbe, mais modalisateur ou adverboïde. Nous proposerons une option différente, qui vise à garder au lexème penser son unité de pleine rection pour l'acception considérée de «estimer» : le verbe se construit avec un déterminant; il sélectionne comme déterminant une séquence représentant un jugement, un procès/processus qui nécessite une relation de prédication. Cependant, il est loisible à l'énonciateur de construire son énoncé autrement, et de considérer non pas que le verbe est un adverboïde, mais que le discours citant n'est pas structurellement matrice mais sousphrase dans l'énoncé, comme dans le cas de l'incise (exemple 4). Dans le cas qui nous occupe ici, l'énonciateur pourrait construire son énoncé en évitant la lecture-analyse de l'exemple 2 (avec la sous-phrase de discours cité comme déterminant du verbe). Il s'agirait plutôt de considérer que la sous-phrase de discours cité est en position de prédicat d'une structure incomplète (sans verbe, sans noyau de phrase), du type « [l y a] que Pierre est déterminé ». On aurait dès lors une sous-phrase prédicat, reliée par prédication première à un noyau zéro. Sur cette relation prédicative échoirait une sousphrase déterminant de l'énonciation (je pense). En schéma:

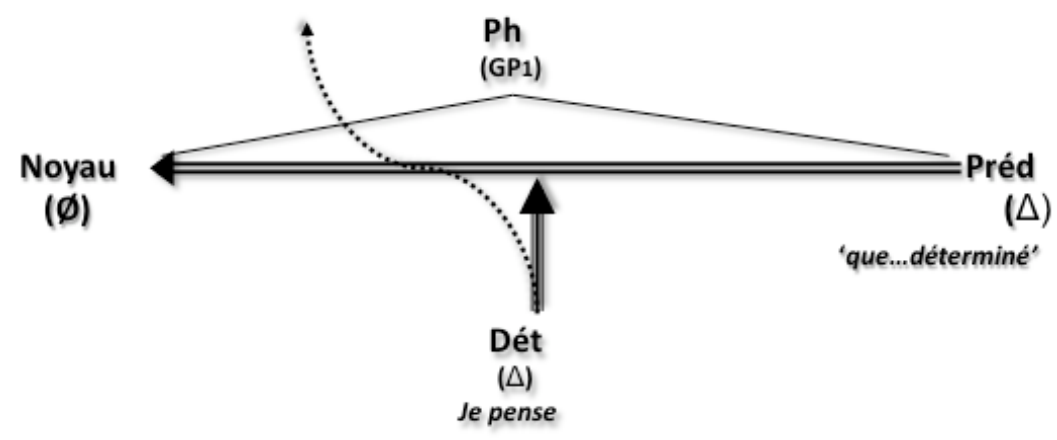

De la sorte nous rendons compte de la possible interprétation de je pense comme modalisateur, non pas parce que l'on aurait affaire à un adverboïde, mais parce que la sous-phrase de discours citant occupe une fonction de déterminant de relation prédicative, avec portée sur la composante énonciation (cadrant le Qui pense?), fonction généralement prise en charge par des adverbiaux. Le verbe ne change pas de nature. La sous-phrase dont il fait partie occupe une fonction prototypiquement considérée comme adverbiale.

Dans ce cadre, le verbe penser voit sa valence complétive non saturée : il ne régit aucun déterminant. Pour autant, on ne parlera pas de rection faible, étiquette d'habitude généralisée à l'ensemble des emplois du verbe ; on parlera de construction (non saturation du déterminant) du verbe en fonction de l'emploi voulu par l'énonciateur.

Une telle analyse pourrait également expliquer des exemples comme Heureusement que Pierre est déterminé, où la sous-phrase que Pierre est déterminé occuperait les mêmes position et fonction que ci-dessus. Heureusement serait quant à lui un GDAdv prédicat second de la relation prédicative. On pourrait imaginer le même schéma encore, mais 
avec un déterminant de l'énoncé cette fois, pour l'exemple typique de subordination inverse : Il n'était pas parti depuis deux minutes que le téléphone sonna. Il n'était pas parti depuis deux minutes serait une sous-phrase déterminant de la relation prédicative entre le prédicat saturé par la sous-phrase que le téléphone sonna et le noyau de phrase zéro.

\subsection{Pierre est déterminé, je (le) pense}

L'analyse précédente de la sous-phrase de discours citant comme déterminant de l'énonciation peut également servir pour décrire cet exemple, où la matrice serait constituée du jugement Pierre est déterminé (non considéré comme un discours reproduit, comme à l'exemple 4). Dans ce cas, la présence du pronom le est possible, qui sature la position de déterminant du verbe, dès lors recteur. En schéma :

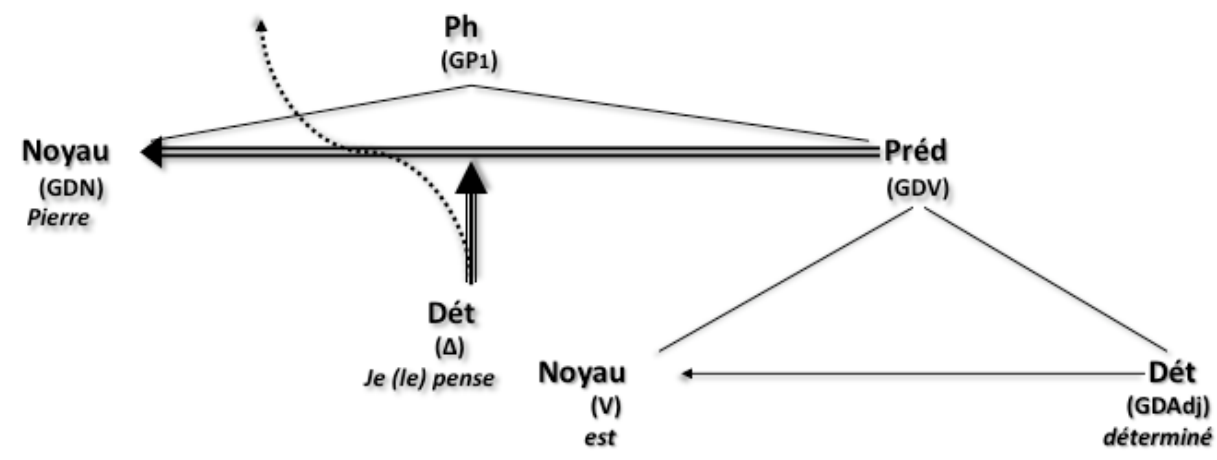

\subsection{Pierre est déterminé, je le pense}

Rien n'empêche toutefois, avec une mélodie adéquate, de rendre compte de cette séquence comme d'une suite de deux prédications formant un tout (une phrase multiple) par juxtaposition, c'est-à dire sans hiérarchisation aucune. Penser régit ici encore le pronom neutre le. Tout dépend donc du vouloir-dire de l'énonciateur. En schéma :

Ph

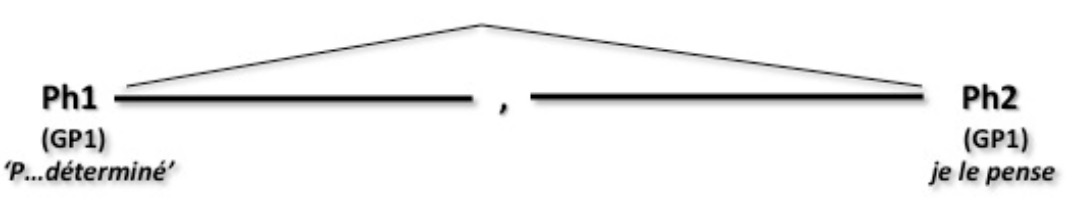

\subsection{Oui, je (le) pense}

Dans la continuité de l'exemple 10, une séquence comme Oui, je pense pourrait être analysée d'une façon assez proche de celle de l'exemple 4. On aurait là une phrase à prédication impliquée Oui, dont l'énonciation serait déterminée par la sous-phrase je 
pense. La présence du pronom le ne semble pas impossible. On rejoint encore une fois les cas où la fonction de la sous-phrase est prototypiquement celle des adverbiaux. Ce qui explique sans doute l'effet modalisateur que l'on impute au verbe. En schéma :

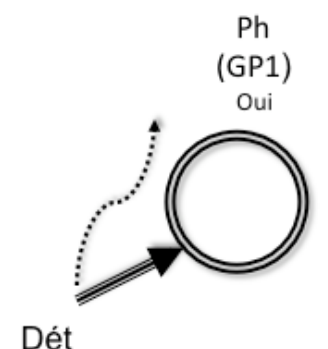

$(\Delta)$

Je pense

\section{Conclusion}

Nous avons proposé d'analyser différentes structures intégrant le pré-dicteur penser dans le modèle que nous avons élaboré. Notre optique était essentiellement syntaxique. Ce faisant, nous avons pu expliquer que la faiblesse de rection, attachée d'ordinaire globalement aux verbes envisagés, tenait moins de leur valeur lexicale de base que des emplois particuliers dans lesquels l'énonciateur avait choisi de les utiliser. Il nous semble en effet difficile de dire que tel verbe qui pourrait se trouver dans trois contextes syntaxiques différents (les trois contextes de base de Blanche-Benveniste \& Willems 2007) serait automatiquement recteur faible pour tous ses emplois : ses modes de rections diffèrent en fait selon les emplois, même si sa valence peut fondamentalement rester la même. Nous avons vu que dans de nombreux exemples, le verbe régissait un déterminant. Dès lors parler de faiblesse de manière généralisée semble abusif. Il semble plutôt qu'il faille traiter la construction de ces verbes en fonction du vouloirdire de l'énonciateur, qui seul est habilité à hiérarchiser l'information comme il le souhaite. Il a à sa disposition plusieurs modes de liaison de prédications, qui peuvent lui permettre, pour une même séquence, d'inverser les rapports matrice/sous-phrase, discours citant/discours cité. Au point d'amener le discours citant à endosser une fonction prototypiquement adverbiale de déterminant de l'énonciation, et d'en perdre la traditionnelle saturation de la position de déterminant du verbe, qui reste, malgré tout, verbe. 


\section{RÉFÉRENCES BIBLIOGRAPHIQUES}

Apothéloz, D. (2003), "La rection dite 'faible': grammaticalisation ou différentiel de grammaticité ?", Verbum, n²5/3, pp. 241-262.

Blanche-Benveniste, C. (1989), "Constructions verbales en incise et rection faible des verbes ", Recherches sur le français parlé, $\mathrm{n}^{\circ}$, pp. 53-73.

Blanche-Benveniste, C. (2001), "Terminologie de quelques relations syntaxiques du domaine verbal : rection, valence, réalisation zéro », in Colombat B. \& Savelli M. (éds), Métalangage et terminologie linguistique, Louvain, Peeters coll. «Orbis Supplementa 17 », pp. 51-64.

Blanche-Benveniste, C. \& Willems, D. (2007), « Un nouveau regard sur les verbes faibles ». Bulletin de la société linguistique de Paris, n¹02/1, pp. 217-254.

ERIKSSON, O. (1993), La phrase française: essai d'un inventaire de ses constituants syntaxiques, Göteborg, Acta Universitatis Gothoburgensis.

GACHET, F. (à par.), "L’incise de discours rapporté : une principale d'arrière-plan ? ", in Defrancq B. et al. (éds), Actes du colloque international Discours et Grammaire 2008, Gand (23-24 mai 2008).

HANON, S. (1989), Les constructions absolues en français moderne, Louvain/Paris, Peeters.

JESPERSEN, O. (1924), The philosophy of grammar, Chicago, University of Chicago press.

LAZARD, G. (1994), L'actance, Paris, PUF.

LAZARD, G. (1998), «Définition des actants dans les langues européennes », in Feuillet J. (éd.), Actance et valence dans les langues de l'Europe, Berlin-New York, Mouton de Gruyter, pp. 11146.

Rosier L. (1999), Le discours rapporté. Histoire, théories, pratiques, Paris/Bruxelles, Duculot.

TESNIÈRE, L. (1965²), Éléments de syntaxe structurale, Paris, Klincksieck.

VAn RaEmdoncK, D. (1998), «Sous mon arbre, volait un esthète », in Annick Englebert, A., Michel Pierrard, M, Rosier, L. et Van Raemdonck, D. (éds): La Ligne claire. De la linguistique à la grammaire. Mélanges offerts à Marc Wilmet, Louvain-la Neuve, Duculot, pp. 237-252.

Van Raemdonck, D. (1998), "Prédication vs détermination: l'exemple de la fonction adverbiale », dans Forsgren, M., Jonasson, K., et Kronning, H. (éds), Prédication, assertion, information. Actes du Colloque d'Uppsala en linguistique française (6-9 juin 1996), Uppsala, Acta universitatis upsaliensis, pp. 579-585.

VAn RAEmDonCK, D. (2001), « De l'incidence comme critère organisationnel du système fonctionnel du français », in De Carvalho, P., Quayle, N., Rosier, L. et Soutet, O. (éds), La Psychomécanique aujourd'bui. Actes du VIIIe Colloque International de Psychomécanique du langage (Seyssel-Chambéry, 19-21 juin 1997), Paris, Champion, pp. 323-341.

VAn Raemdonck, D. (2002), «Discours rapporté et frontières de phrase : l'épreuve de l'intégration syntaxique », Faits de Langues, n¹9, pp. 171-178. 
" Je pense donc ... je dis. » Pour une syntaxe des (pré-) dicteurs

VAN RAEMdonCK, D. (2003), « De la syntaxe incidentielle à l'interprétation pragmatique : le cas de la négation ", in Ouattara, A. (éd.) Parcours énonciatifs et parcours interprétatifs: Théories et applications, Paris, Ophrys, pp. 57-68.

VAN RaEmdoncK, D. (2004), «Discours rapporté et intégration syntaxique: un exemple d'analyse » in Lopez Munoz, J. M., Marnette, S., et Rosier, L. (éds) Le discours rapporté dans tous ses états : question de frontières, Paris, L'Harmattan, pp. 531-537.

VAN RAEMDONCK, D. (2007), «Syntaxe de la phrase et structures intégratives : l'exemple par T et $\Theta$ ", in Brès, $\mathrm{J}$ et al. (éds), Psychomécanique du langage et linguistiques cognitives. Actes du XIe Colloque international de l'Association internationale de Psychomécanique du langage, (Montpellier 8-10 juin 2006), Limoges, Lambert-Lucas, pp. 311-320.

VAN RAEMDONCK, D. (2010), « La rection verbale à l'aune de l'incidence : L'incidence autour et alentour du verbe », Travaux de Linguistique, n60, pp. 45-64.

Wilmet, M. (1986), La détermination nominale, Paris, PUF.

WiLMET, M. (1997, 20105). Grammaire critique du français, Bruxelles, Duculot. 